\title{
ARE YOU TALKING TO ME? WHY BIM ALONE IS NOT THE ANSWER
}

\section{DOMINIK HOLZER}

Spatial Information Architecture Laboratory

School of Architecture and Design

Royal Melbourne Institute of Technology University

GPO Box 2476V, Melbourne, VIC 3001, Australia

Dominik.holzer@rmit.edu.au

\begin{abstract}
In contemporary building practice, the hegemony of 2D-based design communication is gradually being challenged by the possibilities offered by integrated 3D design environments and digital interfaces. The upcoming application of building information modeling (BIM) offers a way out of the current Babylonian plurality of non-compatible modeling-languages in order to push software developers and users to convert towards one common industry standard for data exchange. It is a clear aim of those propagating the use of BIM to strengthen the interaction of design teams and to assist facilities management through common standards for increased interoperability and data-management from the early design stage to completion and operation of a building. Current BIM capabilities rather seem to lie in the area of design documentation and post-design rationalization than triggering new design solutions. This paper sheds light on the status-quo of BIM and questions how designers can complement the current BIM capabilities to increase design-communication and a more seamless flow of information between various parties in architecture, engineering and construction (AEC).
\end{abstract}

Keywords: BIM, Parametric Design, Design Heuristics, Transdisciplinary Design.

\section{Introduction}

Building Information Modelling (BIM) as we know it today has been around in the AEC industry for decades and it has only recently come out of the 'building science' closet. After spending years on the back seat of architectural practice, BIM is suddenly resounding throughout the land and one won't easily find an architecture conference, engineering function or building manager's journal where the topic is not discussed in one way or the other. This step towards becoming 'mainstream' has occurred due to a strong push during recent years from the leading software developers which promote products such as REVIT, TRIFORMA, DIGITAL PROJECTS and ARCHICAD.

Whilst the application of BIM becomes more accepted and widespread throughout the industry, we do find ourselves in the paradox situation that nobody quite seems to agree on how to define BIM anymore. Depending on the sources one will find definitions describing it as method for managing project information where non-geometry attributes get associated with geometrical 
entities, or definitions which mostly point out its capabilities for cost-control and to facilitiesmanagement.

In this paper, the reasons for the current perplexity in defining BIM get analysed and arguments of BIM supporters and antagonists get brought into perspective for a more subtle view on the issue. Further, the author proposes ways out of the deadlock to pour oil on the troubled water surrounding the BIM debate.

\section{Background to BIM}

Before the currently accepted acronym 'BIM' was coined by Autodesk in the first years of the $21^{\text {st }}$ century, a vast development had taken place resulting in a variety of proposals for objectorientated building design linked through commonly accessible databases. Over the past 35 years various investigations for finding methods and tools for computationally sponsored exchange of building-specific information and multi-disciplinary interoperability has been made in the field of Building Science. The predominant aim of this quest was to find ways to organise data-models which allow for a complete integration of all relevant factors in the building lifecycle. As Eastman points out (Eastman, C. 1999 - Building Product Models), all phases in a building lifecycle starting from a pre-design phase of feasibility studies, then design, construction planning, construction, facility management and operation can get described as one holistic process. (Eastman, 42)

A plurality of approaches for knowledge-based design systems which have dealt with similar issues as the current BIM platform since the late 1970s can be found at Khemlani et al (52). The authors discuss inter alia how STEP, the international Standard for the Exchange of Product Model Data (without specific focus on the building sector) provides a basis for the exchange format of BIM models. The International Alliance for Interoperability (IAI) was founded in 1994 and is developing a data-exchange system based on the 'building product model' (Malkawi, A.M. 2005). The data-exchange system consists of a component-based data library with descriptions of building parts and their interrelation in standardised classes - the so called Industry foundation classes (IFC).

In the late 70 s and early 80 s, at a time pre-dating personal computing, the Cambridgedeveloped and commercially applied RUCAPS was using very similar methods for connecting building-design information to those currently set for BIM. RUCAPS operated in a partly parametric environment where 2D information was extracted from a 3D model. It allowed multiuser access and did not mimic common drafting processes, but proposed a novel way to generated, distribute, and retrieve building information. The disadvantages of this early 3D system lay in its inflexibility to produce complex geometrical shapes and the high cost and slow speed of the system. (Day - paragraph 11))

Some of the principal ideas from RUCAPS can be found in current BIM software such as Autodesk's REVIT, Bentley's TRIFORMA, ARCHICAD as well as Gehry Tech's DIGITAL PROJECT.

\subsection{BIM - many definitions- many interpretations}

Due to current marketing-strategies of BIM software providers, it has become difficult to get a distinct picture of the aspects which can be addressed through BIM. These software providers want to make us believe that all aspects of the use of computers in design from early stages to completion and operation can be solved using their specific tool.

When looking at the development of what we currently understand as BIM, there is no indication that it is synonymous for one, all encompassing software solution. One scope for BIM is the seamless integration of information through a standardized format. At the current state of development this implies the capability to bridge the interoperability-gap that exists between distinct software tools. The tasks that are performed in this context vary to a large degree. BIM assists in design documentation, virtual pre-assembly, cost control, construction sequencing and facilities management - just to name a few. Davies argues the notion presented by some providers that BIM has to use 3D building components throughout the whole building-lifecycle is not correct (Davies, paragraph 7). Although BIM might offer possibilities to integrate those, it is traditionally a far more open platform where individual contributors can share information in a standardised format to manage project information.

The point is being made by those propagating the use of BIM that designers can get a better understanding of complex design-issues and resolve them quicker - which - in return - gives 
them more time to focus on design. If the tools currently supporting BIM rather address issues of virtual pre-assembly, error checking, on-site construction coordination and building maintenance, does the benefit for the user then not mainly lie elsewhere than in design?

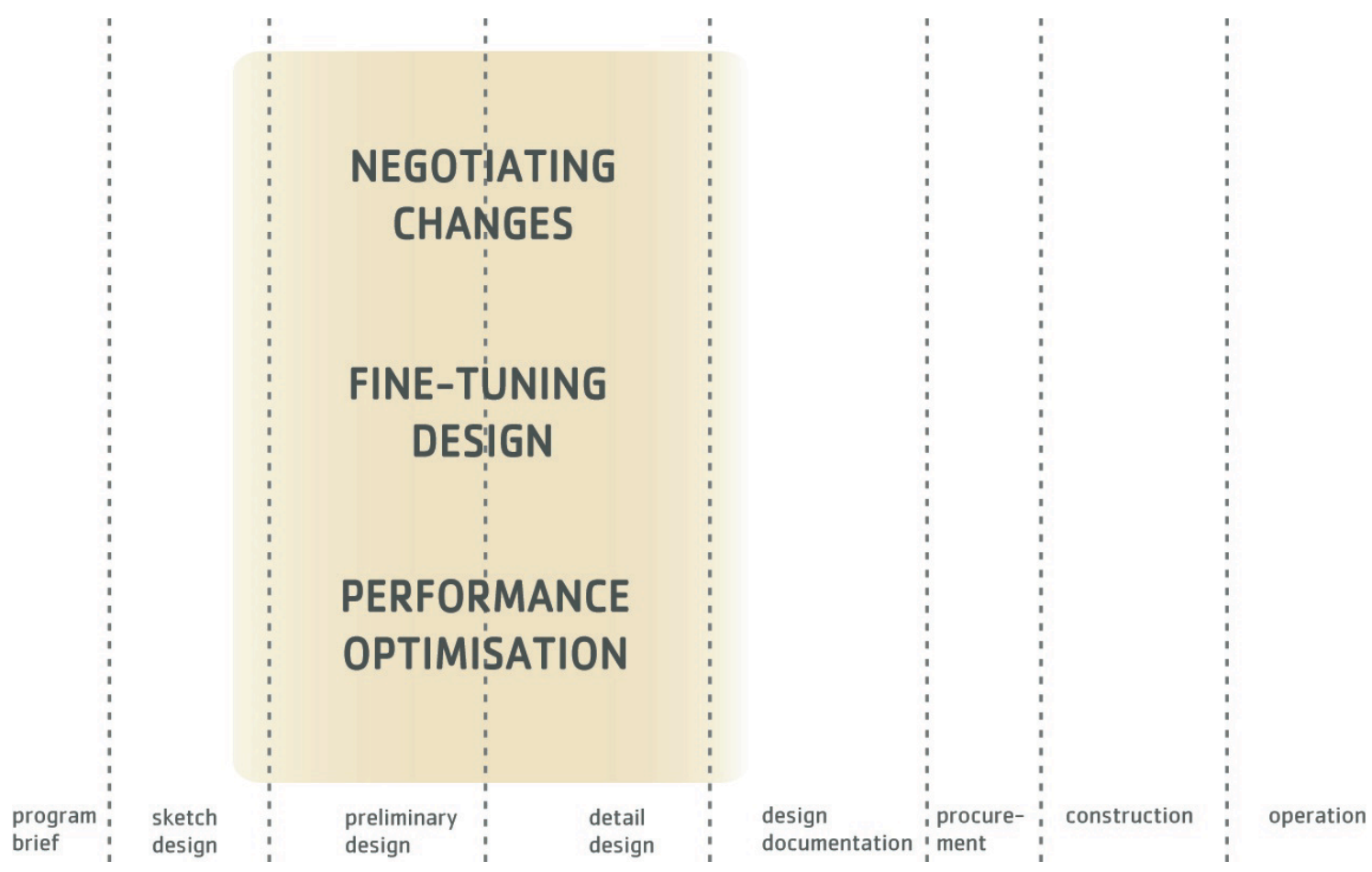

Table 1: BIM capabilities - strength in documentation, construction an operation

\subsection{Who does the work - who takes the profit?}

Those promoting BIM from argue that all involved in a building project will profit from its implementation. The list of possible beneficiaries is extensive and includes clients, architects, engineers, estimators, specifies, contractors, lawyers, sub-contractors, fabricators, code officials and operators - just to name a few (National Institute of Building Science, paragraph 4). The often un-answered question is the one about who actually produces the BIM model and who controls it. The current notion is that the architect - in his/her traditional role as projectcoordinator should be the one to do the work. There does not seem to be a clear indication that this reflects reality on a global level. The distribution of roles is much rather dependant on the level of BIM skills and contractual frameworks in individual countries. A certain reluctance to do the BIM work is apparent amongst architects as they often do not see the advantages it may bring to their work

One reason for this is that architects are by far not the only party who might profit from the creation of a BIM model and it may raise questions in how far it would be their 'job' to produce work which is used for building information management rather than modelling. The work is done by the upstream parties but mainly favors the downstream parties - notably the client, the sub-contractors and the operators. The model becomes a digital asset, but the added value is currently not understood by all parties involved in the BIM process.

We need more transactional compensation models to re-allocate the financial benefits to those who do the work. This issue has been discussed by Aish in reflection on the failure of the above mentioned RUCAPS and the lessons we can learn for the present implementation of BIM (Aish, 2). Once contractual issues about compensation as well as responsibilities and liabilities of individual parties for work undertaken on BIM are agreed on, who is supervising the process of collating all the information that feeds into the integrated model? Ceccato encourages architects to take advantage of the shift which is currently occurring in the building industry to regain lost ground in the design and building process through a redefinition of their professional status as integrators (Ceccato, paragraph 8). The person who is responsible to coordinate the BIM data 
will have to be able to capture design intent and expert knowledge to encode and incorporate it to the information platform next to the geometry-data which is provided by individual parties.

\subsection{Outside pressures - BIM as legal requirement}

The US General Services Administration (GSA) has issued a BIM-guideline in late 2006 which introduces their roadmap for a stronger integration of the use of BIM in the US AEC sector in general and the Public Building Service (PBS) in particular. The point is made that the GSA has instantiated a requirement in 2007 which will force all planners to produce BIM models for spatial program validation as an open standard if they apply for funding for their projects (GSA, 14). This is a significant shift for a public client to pro-actively push the industry to use a specific standard - a method which has now also become a legal requirement in Denmark since the beginning of 2007 .

The issue of code-compliance might represent a further incentive to design using BIM. 3D integrated models are a far more accurate representation of any building project than the currently used 2D plan material. It is likely that in not too far future, BIM models will become more convincing instruments in communicating design and get it signed off by the authorities

\subsection{BIM: A framework for design?}

Those in favour of the implementation of BIM advocate its usefulness in bridging design intelligence across disciplines from pre-design to operation. How can this be instantiated? As much as the BIM approach allows for better data-transfer and integration, it currently does not entirely seem to encompass and link into processes that occur in the creative, conceptual design phases.

Lawson argues that the way CAD in general is currently implemented in design, planning and construction, falls short of supporting the design process in favour of assisting in drafting and documentation. The reciprocal feedback processes which occur between pencil and brain during conceptual design have not been mirrored with according CAD tools. Lawson draws a distinction between design as a problem-solving activity and an act where designers apply semantic and episodic knowledge to develop solutions through experiential memory. In case of the latter, designers combine slow reflection with the necessity to keep many things in mind at the same time for rapid decision making (Lawson, "Oracles" 389). Current tools often do not allow designers to keep various options open for evaluation simultaneously. What does this mean for BIM?

With current computational capabilities, there is a computational limitation in the design aspects that can be addressed parametrically. The One Island East project in Hong Kong developed by Swire is one of the first office buildings that have been pre-designed virtually in the parametric BIM software Digital Project (DP) by assembling up to 300.000 building components in one master-file. As much as DP is fully enabling parametric design, it was only used for digital preassembly, integration of building components, clash detection, construction sequencing and facilities management. The design was not set up parametrically due to the enormous complexity of handling the amount of detailed building information implicit in 3D design documentation. The more information is added into a BIM model, the less likely one will be able to remain flexible in the creation of alternative versions. This leads to the assumption that with current computational capabilities, a BIM model which includes the necessary information for documentation only makes sense once the basic geometry remains fixed and will not be altered. For design-explorations in the earlier design stages where changes occur due to the input of a variety of reasons, lighter data-sets and models for project-representations are required.

The question thus remains: How far down the track in the design process should we start using BIM? Can one single BIM model assist in the design process from the early stages to operation and demolition?

\section{Complementing the BIM process}

There is not question about the usefulness of BIM in regard to data-interoperability issues, but rather about the nature of support it can/should offer in the various design stages. In how far is there a limit to what digital representations can do and where human interaction and communication together with new skill sets are required? If dealing with uncertainty is seen as an intrinsic quality for creative processes, how can we avoid that standardised computational frameworks obstruct the design process rather than supporting it? What instruments do we have 
at hand for the design stages where we are not yet working with detailed descriptions of building objects, but want to evaluate options and keep the design flexible enough for input form design intelligence coming from our partners?

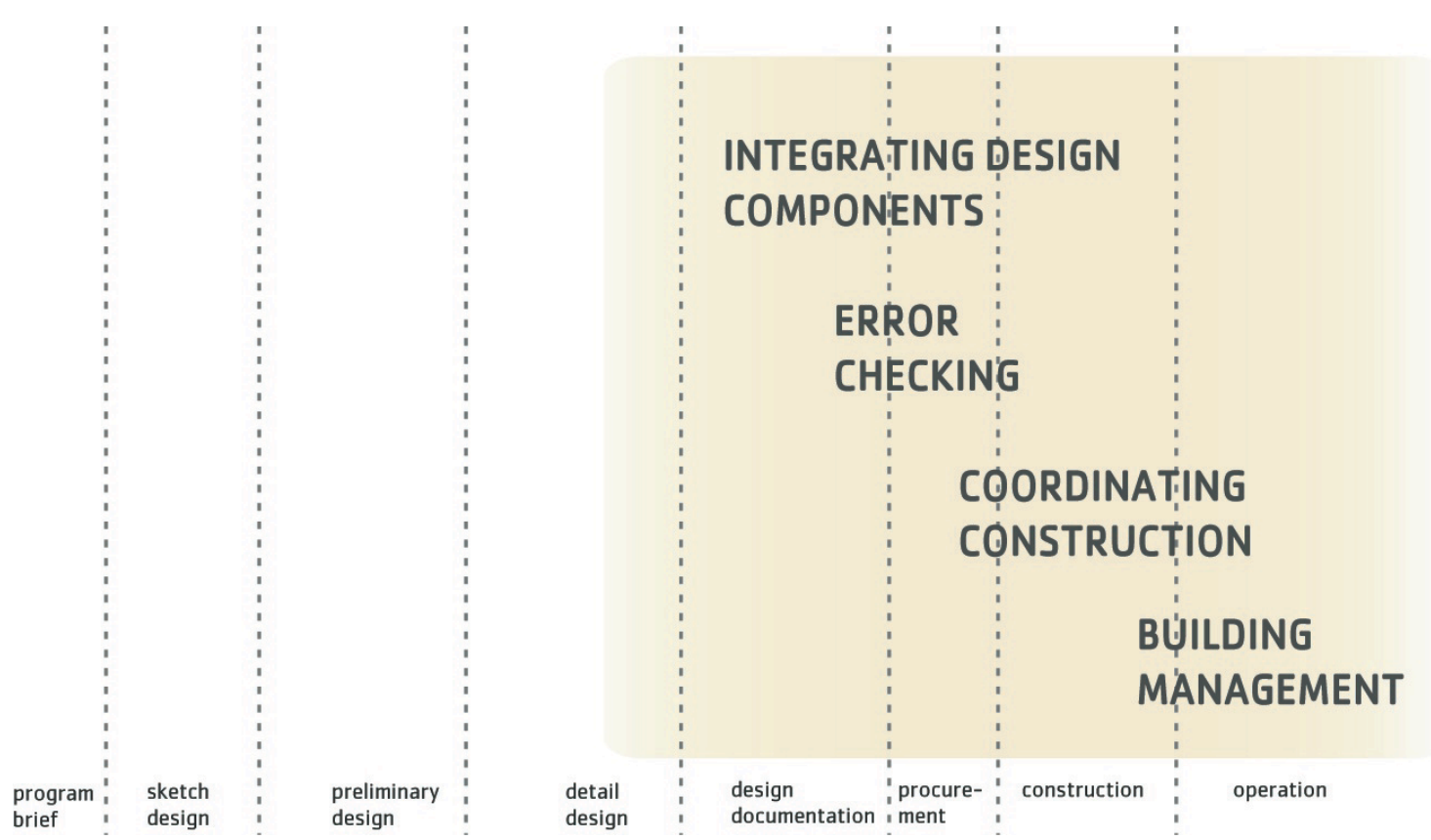

Table 2: Flexible design in earlier stages, creating versions - evaluating options

\subsection{Analysing the process, finding toolsets for design decision support}

We need to complement the possibilities of data-exchange with design methodologies that sponsor knowledge transfer across teams. In particular in the early design stages, where we are dealing with a high degree of uncertainty we require support to define, weight, and prioritise aspects of design in collaborative processes.

Can processes which lead to 'good design' be captured and replicated to create process maps for increased efficiency? After 25 years of in-depth observation of the way designers work Lawson concludes that a shared view of the design process is more myth than reality. Lawson further asserts that it is unlikely that efficiency of process would automatically equate with better design (Lawson, "Designers" 258). There exists an apparent antagonism between these comments and the claims most BIM supporters raise. Evidence suggests that under certain conditions, BIM systems can be augmented by manuals that describe and display information for construction of building-projects in the shape of process-maps (Mitchell, Wong, and Plume 321). These Information Delivery Manuals (IDM) have originated in Norway and they serve as framework for multi-disciplinary teams to improve communication amongst various teams in the construction process. IDMs provide assistance for BIM users by using descriptions of building construction processes as well as support for BIM software providers by identifying and describing detailed functional breakdowns of the process to be supported. When developing process models, how can we agree on the importance of each contribution by the individual members of the design team?

Deiman and Plat argue that referencing information about cost-consequences to designdecisions is a key factor for evaluating their importance in succeeding stages. The earlier decisions are made in the design process, the more significant is their impact on the final outcome. Deiman and Plat hence propose applying different levels of granularity of datarepresenting for building projects by clustering elements into design parts (Deiman and Plat 328). In accordance with this basic concept, the research of Khajehpour and Grierson illustrates an example of evolutionary search algorithms together with Pareto optimisation can assist in finding trade-offs between lifecycle profitability and structural considerations in a highrise project. Multi-objective design optimisation is carried out in the early design stages by first assembling quantitative data about capital cost, operating cost and income revenue over time. 
This gets brought into relation with quantitative data about building typology, structural systems, material usage, transport costs etc. A relational matrix of design aspects influencing cost and revenue considerations is established and consequently a set of alternative designs for the building is created (Khajehpour and Grierson 281). The aim in this multi-objective optimisation process is not to find one optimum solution, but to offer designers an array of designs which represent a good compromise between profitability and structural safety. This high-lightens that there are computational methods that can assist in providing effective decision support for solving complex multi-objective design problems. At the same time, it shows that expert input for prioritising one solution to another is always required and that the computational assistance can offer a greater variety of informed solutions to choose from.

\subsection{Stepping outside one's own domain}

Once individual users or user groups have developed their own working method they can enter a wider dialogue with others and to take a simple step at a time. In order to work towards integrated practice we require an intensive dialogue with the end parties who receive our information to understand their work methodology, skill sets and the way they interface data (be it for design, analysis or production). This will enable us to 'work backwards' to inform our own design-processes towards integrated practice.

Designers are often not aware of the interfacing potential between the information they produce, and the information that is needed to drive manufacturing processes. It has shown beneficial for designers to be aware of constraints and possibilities of the building-manufacturing industry as early as possible in the planning process. In consideration of project to project specificity, advantages can be gained through designated interconnections with the manufacturers and contractors to gain knowledge about their work-methodologies, their production constraints and their digital interfaces. In some cases, simple custom-made scripts and programming interfaces can create seamless CAD/CAM links from design to production. Only if we demonstrate the benefits of this rationalisation to the client and the quantity surveyor, we can prove that cost can be reduced to increase feasibility.

Alternatives to current tendering processes can assist designers to share the benefits and the risk of their work with the sub-contractors. Tombesi has investigated the 'request for proposal' method as practiced in Frank Gehry's office as a new way of organising tenders for subcontractors (Tombesi 86). When using request for proposal tenders, a 3D information package is distributed amongst competing subcontractors at a stage where no contract is signed yet; no detail drawings are made available to them, but accurate design intent - flexible about engineering strategies.

\section{Conclusions}

BIM is a useful way of increasing interoperability and pushing the agenda of integrated practice throughout the whole AEC industry. Next to that, its usefulness for the clients and operators of buildings is unquestioned and there are indications that its implementation will increasingly become important as a legal requirement. As designers, we have to scrutinise our design methodology to distinguish where BIM can assist us to do our work more efficiently, but also where it might limit our creativity in the conceptual design process. Only by doing so, we can develop our own specific way of designing with the help of computational processes which are currently not supported by BIM.

\section{References}

Aish, Robert. "Migration from an individual to an enterprise computing model and its implications for AEC Research." White paper submitted to the Berkeley-Stanford CE\&M Workshop, (2000): 2

Ceccato, Chris. "Integration: Master [Planner/Programmer/Builder]." International Conference on Generative Art, url:http://www.generativeart.com/ last accessed on 12.04.2005: paragraph 8.

Davies, Nigel. "(Mis)understanding BIM" http://www.eatyourcad.com/article.php?incate_id=14778, accessed on 12.05.2007.

Day, Martyn. "Intelligent Architectural Modeling." AEC Magazine (2002) http://www.caddigest.com/subjects/aec/select/Intelligent_modeling_day.htm accessed on 12.05.2007: paragraph 11. 
Deiman, Erik, Plat, Herman T. "Cost information in succeeding stages of the design process." Advanced Technologies, M.R.Beheshti and K.Zreik (Ed.) B.V.: Elsevier Science Publishers 1993: 327.

Eastan, Chuck. Building Product Models. Florida: CRC Press LLC, 2000.

GSA 01 - US General Services Administration, BIM Guide Overview Version 0.50 url: www.gsa.gov/gsa/cm_attachments/GSA_DOCUMENT/GSA_BIM_02_Main_v09_R2C-a3I_0Z5RDZ-i34K-pR.pdf accessed on 18.05.2007 (2006): 14.

Khajehpour, Siavash and Grierson, Don E. "Profitability versus safety of high-rise office buildings." Structural Multidisciplinary Optimisation 25, Springer Berlin, DOI 10.1007/s00158003-0297-4, (2003): 279.

Khemlani, Lachmi, Timerman, Anne, Benne, Beatrice and Kalay, Yehuda E. "Intelligent Representation for Computer-Aided Building Design." Automation in Construction 8 (1998): 52.

Lawson, Bryan. "Oracles, Draughtsmen, and Agents: the nature of knowledge and creativity in design and the role of IT." Automation in Construction 14 (2005): 389.

Lawson, Bryan. How Designers Think. Oxford: Elsevier Architectural Press, 2006: 258.

Malkawi, Ali M. Performative Architecture: Beyond Instrumentality. 2005: 233.

Mitchell, John, Wong, Justin and Plume, Jim. "Design Collaboration Using IFC.” Proceedings of the 12th International CAAD Futures Conference Dong, Andy; Moere, Vande, Andrew; Gero, John S. Eds. 2007: 317.

National Institute of Building Science. About the National BIM Standard. http://www.facilityinformationcouncil.org/bim/about.php accessed on 11.05.2007: paragraph 4.

Tombesi, Paolo. “Involving the industry.” Architectural Research Quarterly 6 (1) (2002): 86. 